\title{
Consensus of the Hellenic Headache Society on the diagnosis and treatment of migraine
}

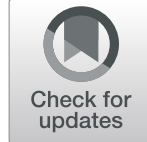

\author{
Evangelos Kouremenos ${ }^{1}$, Chrysa Arvaniti ${ }^{2}$, Theodoros S. Constantinidis ${ }^{3}$, Ermioni Giannouli ${ }^{4}$, Nikolaos Fakas ${ }^{5}$, \\ Themistoklis Kalamatas ${ }^{4}$, Evangelia Kararizou ${ }^{6}$, Dimitrios Naoumis ${ }^{1}$, Dimos D. Mitsikostas ${ }^{6 *}$ and Hellenic Headache Society
}

\begin{abstract}
More than 0.6 million people suffer from disabling migraines in Greece causing a dramatic work loss, but only a small proportion of migraineurs attend headache centres, most of them being treated by non-experts. On behalf of the Hellenic Headache Society, we report here a consensus on the diagnosis and treatment of adult migraine that is based on the recent guidelines of the European Headache Federation, on the principles of Good Clinical Practice and on the Greek regulatory affairs. The purposes are three-fold: (1) to increase awareness for migraine in Greece; (2) to support Greek practitioners who are treating migraineurs; and (3) to help Greek migraineurs to get the most appropriate treatment. For mild migraine, symptomatic treatment with high dose simple analgesics is suggested, while for moderate to severe migraines triptans or non-steroidal anti-inflammatory drugs, or both, should be administered following an individually tailored therapeutic strategy. A rescue acute treatment option should always be advised. For episodic migraine prevention, metoprolol (50-200 mg/d), propranolol (40-240 mg/d), flunarizine (5$10 \mathrm{mg} / \mathrm{d})$, valproate $(500-1800 \mathrm{mg} / \mathrm{d})$, topiramate $(25-100 \mathrm{mg} / \mathrm{d})$ and candesartan $(16-32 \mathrm{mg} / \mathrm{d})$ are the drugs of first choice. For chronic migraine prevention topiramate $(100-200 \mathrm{mg} / \mathrm{d})$, valproate $(500-1800 \mathrm{mg} / \mathrm{d})$, flunarizine (5$10 \mathrm{mg} / \mathrm{d})$ and venlafaxine $(150 \mathrm{mg} / \mathrm{d})$ may be used, but the evidence is very limited. Botulinum toxin type A and monoclonal antibodies targeting the CGRP pathway (anti-CGRP mAbs) are recommended for patients suffering from chronic migraine (with or without medication overuse) who failed or did not tolerate two previous treatments. Anti-CGRP mAbs are also suggested for patients suffering from high frequency episodic migraine ( $\geq 8$ migraine days per month and less than 14) who failed or did not tolerate two previous treatments.
\end{abstract}

Keywords: Hellenic headache society, Consensus, Migraine, Treatment

\section{Introduction}

Migraine is the third most prevalent disorder in the world [1] and the first cause of disability in under 50s [2]. Almost three billion individuals were estimated to have migraine or tension-type headache (TTH) in 2016 (1.89 billion with tension-type headache and 1.04 billion with migraine). However, because migraine has a much higher disability burden than TTH, migraine caused $45 \cdot 1$ million (95\% UI 29.0-62.8) and TTH only 7.2 million (95\% UI 4.6-10.5) YLDs (Years of Live lived with Disability) globally in 2016 [1]. In Europe, the mean perperson annual costs were $€ 1222$ for migraine and $€ 3561$

\footnotetext{
* Correspondence: dmitsikostas@med.uoa.gr

${ }^{6}$ First Neurology Department, School of Medicine, National \& Kapodistrian University of Athens, Aeginition Hospital, 72-74 VI Sofia's Avenue, 11528 Athens, Greece

Full list of author information is available at the end of the article
}

for medication-overuse headache, that usually comorbid with chronic migraine, indicating that migraine is a prominent health-related driver of economic losses for the European Union [3]. In Greece about 610.000 suffer from disabling migraines and 283.500 from disabling TTH. Headache sufferers report headache-induced reductions in performance around 3 days/month and only one out of five seek professional treatment for headaches, a private neurologist most often. Interestingly, the vast majority of Greek headache sufferers have never taken prophylaxis for headaches [4]. These findings prompted Hellenic Headache Society (HHS) to initiate a network of headache centres that should manage headache sufferers appropriately. In this light, the consensus outlined here represents an additional effort of the HHS to provide a practical tool including both established and novel treatments for headache and migraine in 
particular, which can easily be used by Neurologists, General Practitioners and any other health professional involved in headache medicine.

\section{Methods}

The present consensus was drafted by a group of members of the Hellenic Headache Society, in three consecutive meetings followed by four email rounds to establish the final document which was approved by all members of the panel. The recommendations of the European Headache Federation (EHF) for technical investigation for primary headache disorders [5] and for treatment of migraine $[6,7]$ were the bases for adaption into the national environment. The European Federation of Neurological Societies (EFNS) recommendations for migraine [8] and medication overuse headache [9] were reviewed and additional literature search was performed for updating the treatments, following the methodology described by EFNS task forces. The patients' preferences obtained in a previous study performed by the HHS [10] were also taken into consideration, together with the National Pharmacy Affairs rules for treatment reimbursement. Migraine diagnosis and classification follows ICHD-3 [11].

\section{Migraine diagnosis}

The diagnosis of migraine is based on the history given by the patient, as well as the normal clinical examination. Only in selected cases is brain imaging required (Table 1) [5]. As no biomarkers exist, taking the patient's medical history remains the most important part of the diagnostic procedure. The presence of particular features often requires paraclinical evaluation to exclude secondary headaches (Table 2). The HHS advises the use of a semi-structured interview to avoid forgetting important information leading to misdiagnosis. An electronic patient interview and database has been developed by the HHS and is available for headache centers and other physicians who treat headaches in Greece to interview and follow up headache sufferers. Briefly, a number of questions, regarding headache onset, duration, localization, severity (rated in a 11 point visual analogue scale-VAS) and frequency, the pain characteristics, the pattern and the possible trigger causes of headache should be routinely asked. Specific scales to scan for potential affective disorders, e.g. the Hamilton depression and anxiety rating scale are significant [12], together with a list of questions related with disorders of the other body systems. Scales for quality of life changes due to headache (e.g. the Migraine Disability Assessment Test- MIDAS [13], the Headache Impact Test-HIT-6 [14]), that also rate the headache impact and severity are recommended for both the first visit and the follow-up. Likewise, a patient's headache diary is of great importance for both diagnosis and follow-up. Headache diary, MIDAS (validated in Greeks [13]), HIT-6 [14] (not validated, translated into Greeks) and Hamilton scales
Table 1 Tests recommended for migraine

\begin{tabular}{|c|c|c|}
\hline ICHD-3 code & Migraine Subtype & Recommended tests \\
\hline \multirow[t]{2}{*}{1.1} & Migraine without aura & None \\
\hline & Frequent Episodic Migraine & $\begin{array}{l}\text { Brain } \mathrm{MRI}^{\mathrm{a}} \\
\text { Carodit ultrasound } \\
\text { or } \mathrm{MRA}^{\mathrm{a}} \\
\mathrm{ESR}^{\mathrm{a}}\end{array}$ \\
\hline 1.2 & Migraine with aura & Brain $\left.M R\right|^{a}$ \\
\hline 1.2 .2 & $\begin{array}{l}\text { Migraine with brainstem } \\
\text { aura }\end{array}$ & $\begin{array}{l}\text { Brain MRI \& MRA } \\
\text { EEG }^{\mathrm{a}} \\
\text { Carotid and vertebral arteries } \\
\text { ultrasound/or CT or MRA } \text { MRA }^{a} \\
\text { Genetic evaluation }^{\text {a }}\end{array}$ \\
\hline 1.2 .3 & Hemiplegic migraine & $\begin{array}{l}\text { Brain MRI \& MRA } \\
\text { Genetic evaluation }\end{array}$ \\
\hline 1.2 .4 & Retinal migraine & $\begin{array}{l}\text { Brain MRI \& MRA } \\
\text { Ophthalmologic evaluation }\end{array}$ \\
\hline 1.3 & Chronic migraine & $\begin{array}{l}\text { Brain MRI Gd \& MRV } \\
\text { Fundoscopy }^{a} \\
\text { Lumbar puncture } \\
\text { Polysomnography }\end{array}$ \\
\hline 1.4 & Complications of migraine & Brain MRI \\
\hline 1.4 .2 & $\begin{array}{l}\text { Persistence aura without } \\
\text { infraction }\end{array}$ & $\begin{array}{l}\text { Emergency brain CT or MRI } \\
\text { (Carotid and vertebral arteries } \\
\text { ultrasound/or CT or MRA ESR }\end{array}$ \\
\hline 1.4 .3 & Migrainous infarction & $\begin{array}{l}\text { Emergency brain CT or MRI } \\
\text { (Carotid and vertebral arteries } \\
\text { ultrasound/or CT or } \mathrm{MRA}^{\mathrm{a})} \\
\text { ESR }^{\mathrm{a}}\end{array}$ \\
\hline 1.4 .4 & $\begin{array}{l}\text { Migraine aura-triggered } \\
\text { seizures }\end{array}$ & Repetitive EEGs or video EEG \\
\hline 1.5 & Probable migraine & Brain $M R I^{a}$ \\
\hline 1.6 & $\begin{array}{l}\text { Episodic syndromes that may } \\
\text { be associated with migraine }\end{array}$ & Gastric work-up \\
\hline
\end{tabular}

Adapted from Mitsikostas et al., 2015 [5] with changes andicates specific conditions; CT Computerized tomography, MRI Magnetic resonance imaging, MRA Magnetic resonance angiography, MRV Magnetic resonance venography, Gd Gadolinium, EEG Electro-encephalogram, ESR Eryhtrocyte sedimentation rate

(validated in Greeks [15]), are all available upon request from the HHS. Apart from the detailed interview the complete neurological examination should be normal. Physical examination, including blood pressure, body mass index calculation and fundoscopy for papilledema are recommended as well.

\section{Treatment of migraine}

The management of migraine is multidisciplinary involving pharmaceutical and non-pharmaceutical procedures that are all important and necessary. Here we report suggestions for the pharmaceutical treatment of migraine that is divided into symptomatic and prophylactic. We also cover the potential use of neurostimulation and food supplements as treatment options. No safety issues are covered but we address, of course, particular considerations when it is appropriately significant. 
Table 2 Clinical features warning of a possible underlying disorder

\author{
- Headache that peaks in severity in less than five minutes \\ - New headache type versus a worsening of a previous headache \\ - Change in previously stable headache pattern \\ (e.g. progressive headache, worsening over weeks or longer) \\ - Headache that changes with posture (e.g. standing up) \\ - Headache awakening the patient \\ - Headache precipitated by physical activity or Valsalva manoeuvre \\ (e.g. coughing, laughing, straining) \\ - First onset $\geq 50$ years of age \\ - Focal neurological symptoms or signs \\ - Trauma \\ - Fever \\ - Seizures \\ - History of malignancy \\ - History of HIV or active infections
}

Adapted from Mitsikostas et al., 2015 [5] with changes

\section{Symptomatic treatment of migraine}

Patients should be advised to receive the symptomatic treatment as soon as they are sure that they are experiencing a migraine attack. Intake of acute medications for migraine must not exceed the 10 days per month for ergotamine, triptans or combinations of drugs, or the 15 days per month for NDAIDs, paracetamol and aspirin, to avoid medication overuse headache. However, we suggest stricter limits to safeguard more (see Medication Overuse Headache Section). Simple analgesics (paracetamol and aspirin, alone, or in combinations, with or without caffeine) are recommended at high doses (e.g. $1 \mathrm{~g}$ of paracetamol, or aspirin, per os) for acute treatment of migraines that are typically mild in severity and duration (e.g. rating less than 6/10 in VAS and lasting less than 12 h) [8]. Before intake of non-steroidal anti-inflammatory drugs (NSAIDs) and triptans, oral metoclopramide or domperidone may be useful when migraineurs experience nausea in particular. For the mild to severe migraine attacks, oral NSAIDs and triptans are suggested [8]. There is good documentation (level A) for naproxen (500-1000 $\mathrm{mg})$, ibuprophen (200-800 mg) and diclofenac (50-100 $\mathrm{mg}$ ), and for tolfenamic acid (200 mg, level B). We do not suggest following the concept of stratified treatment as EFNS Task Force suggests, however [8]. We believe that a therapeutic plan tailored to the individual patient is more efficient, saves time and fits better to the good clinical practice rules. The personal medical history of each patient will guide the physician to the right decision-making. For severe migraine attacks (e.g. menstrual migraine) subcutaneous sumatriptan is recommended.

Headache may recur within 24 or even within $48 \mathrm{~h}$ after successful treatment (meaning pain free $2 \mathrm{~h}$ post treatment) with triptans [16]. One to four out of 10 patients taking an oral triptan experience recurrence [16]. A second dose of the triptan is often effective but only in case the headache recurs. On the contrary, physicians should clarify to their patients that if the first dose of a triptan does not work, a second dose is useless [17]. Interestingly, there is good documentation (level A) that combining a NSAID with a triptan (naproxen $500 \mathrm{mg}$ with sumatriptan $85 \mathrm{mg}$, or sumatriptan $100 \mathrm{mg}$ since in Greece this dosage is not available) reduces headache recurrence and improves efficacy [18-20]. Not all patients are candidates for all of the above treatment suggestions, since there are several contraindications and significant safety issues [21] that this report does not cover (Table 3).

Because the consistency of all available symptomatic treatments is limited, all migraineurs should be advised for rescue treatment. The HHS emphatically recommends

Table 3 Use of simple analgesics, NSAIDs, triptans or combinations for the symptomatic treatment of migraine

- For decision-making physicians should take into account the patients' preferences, life-style particularities and personal expectancies.

- In patients with mild migraines we suggest using aspirin or paracetamol $1 \mathrm{~g}$, per os, for the symptomatic treatment of migraine.

- In patients with migraine who have failed to aspirin or paracetamol 1 g, or who cannot use these agents because of comorbidities, adverse events or poor tolerability, or their migraines have mild to severe intensity, we suggest using triptans per os, or non-steroidal antiinflammatory drugs (NSAIDs), or combinations, according to their potential comorbidity and tolerance to these drugs.

- If migraines recur after successful symptomatic treatment, the use of long acting triptans may be useful (e.g. almotriptan, naratriptan and fravotriptan).

- If migraines do not respond to oral high efficacy triptans (e.g. eletriptan 40 or 80 mg, or rizatriptan $10 \mathrm{mg}$ ) a combination of a triptan with NSAID, sumatriptan $50 \mathrm{mg}$ or $100 \mathrm{mg}$ with naproxen $500 \mathrm{mg}$ in particular, is recommended.

- Not all triptans share the same efficacy, safety and pharmacokinetics within an individual, thus no response to one triptan does not predict unresponsiveness to all triptans.

- Consistency of triptans and other symptomatic drugs for migraine is limited and may vary by individual; therefore, rescue medication should be offered to all patients for symptomatic treatment of migraine; this should be parenterally given to avoid potential gastric stasis, e.g. sumatriptan subcutaneously, or NSAID suppositories.

- All migraineurs should be educated by their treating physicians to limit the use of symptomatic anti-migraine drugs to a maximum of two days per week if they use triptans, or combinations of drugs, or three days per week if they use NSAIDs or simple analgesics, in order to avoid medication overuse headache (since the related ICHD-3 limits are 10 and 15 days/month [11], by this suggestion we prevent from medication overuse securely).

- All migraineurs should be educated by their treating physicians that the symptomatic treatment of migraine is only a small part of the management of migraine, that requires additional prophylactic pharmacotherapy in most cases, life-style changes and nonpharmaceutical approaches depending on the particular patient and the potential comorbidity. 
this in order to limit both the unnecessary hospital admissions and the patients' long suffering. Rescue treatment should be offered when $2 \mathrm{~h}$ post treatment there is no pain relief and should be parenteral for quicker drug absorption and efficacy and because at this late stage of a migraine attack gastric stasis may occur. Apart from subcutaneous sumatriptan, the NSAIDs suppositories are suggested, since no other formulations are currently available in Greece. Intramuscular diclofenac $75 \mathrm{mg}$ may be given as rescue medication at home [22], as well. Status migrainosus can be treated by intravenous cortoicosteroids, although this has not universally been proved to be helpful [8]. Other options include intravenous valproate [23] and a combination of intravenous tramadol with paracetamol [24]. Intravenous metoclopramide alone does not seem to help [25], although there is good evidence for a combination of metoclopramide $10 \mathrm{mg}$ and aspirin $1 \mathrm{~g}$ for the symptomatic treatment of migraine, given per os, at home [26]. Because of lack of evidence demonstrating efficacy and major concern about sub-acute or long-term sequelae, injectable morphine and hydromorphone should best be avoided [27]. Generally, HHS suggests avoiding the use of narcotics for migraine treatment, either for acute or for prophylactic treatment (with the exception of tramadol in a hospital setting, in a few selected cases).

\section{Prophylactic treatment of migraine}

When migraine is disabling and/or the attacks are intractable, prophylactic treatment is suggested. The prevention of migraine chronification is the most important part of migraine management and includes not only the pharmaceutical treatment of migraine but also the treatment of potential comorbidities, such as depression-like conditions and obesity [28]. There is not a cut off threshold of the number of migraine days per month that requires prophylactic treatment. The treating physician decides along with the patient upon the disability and the comorbidities that the specific patient may suffer from. To make a more tailored choice of medication for migraine prevention, HHS suggests the sub-classification of episodic migraine into low (< 8 migraine days per month) and high frequency (814 migraine days per month) episodic migraine. By this sub-classification the new emerging treatments (e.g. the anti-CGRP monoclonal antibodies) fit better to the suggested therapeutic algorithm.

\section{Episodic migraine}

For the prevention of episodic migraine (EM) the HHS accepts the EFNS task force recommendations [8]. The drugs of first choice with level A documentation are metoprolol $(50-200 \mathrm{mg} / \mathrm{d})$, propranolol $(40-240 \mathrm{mg} / \mathrm{d})$, flunarizine $(5-10 \mathrm{mg} / \mathrm{d})$, valproate $(500-1800 \mathrm{mg} / \mathrm{d})$ and topiramate $(25-100 \mathrm{mg} / \mathrm{d})$. Drugs of second choice with level B documentation are amitriptyline $(50-150 \mathrm{mg} / \mathrm{d})$, venlafaxine $(75-150 \mathrm{mg} / \mathrm{d})$, naproxen $(500-1000 \mathrm{mg} / \mathrm{d})$ and new purified formulation of petasites $(150 \mathrm{mg} / \mathrm{d})$. Angiotensin II receptor blocker candesartan (16-32 mg/ d) should be added to the list of first choice medications $[29,30]$. There is margnial documentation for several other agents that have been tested for the prevention of EM (third choice drugs) [8]. For the high frequency EM prevention, HHS follows the EHF recommendations and suggests the use of anti-CGRP monoclonal antibodies (anti-CGRP mAbs) as second choice after the failure of two first choice agents (Table 4).

\section{Chronic migraine}

For the prevention of chronic migraine (CM), HHS suggests the use of first choice drugs recommended for the

Table 4 Use of anti-CGRP monoclonal antibodies for migraine prevention

- In patients with high frequency episodic migraine who have failed at least two of the available medical treatments or who cannot use other preventive treatments because of comorbidities, adverse events or poor compliance, we suggest the use of erenumab, fremanezumab, or galcanezumab;

- In patients with chronic migraine who have failed at least two of the available medical treatments or who cannot use other preventive treatments because of comorbidities, adverse events or poor compliance, we suggest the use of erenumab, fremanezumab, or galcanezumab;

- In patients with high frequency episodic migraine, before starting erenumab, galcanezumab or fremanezumab we suggest to stop oral preventive drugs unless the patient had a previous history of chronic migraine before prevention; in this case, we suggest adding the antiCGRP monoclonal antibody to the ongoing treatment and to re-assess the need of treatment withdrawal;

- In patients with chronic migraine who are on treatment with any oral drug with inadequate treatment response we suggest adding erenumab, fremanezumab, or galcanezumab and considering later withdrawal of the oral drug. In patients with chronic migraine who are on treatment with onabotulinumtoxinA with inadequate treatment response we suggest stopping onabotulinumtoxinA before initiation of erenumab, fremanezumab, or galcanezumab. In patients with chronic migraine who are on treatment with erenumab, fremanezumab, or galcanezumab and who may benefit from additional prevention we suggest adding oral preventive drugs;

- In patients with high frequency episodic or chronic migraine, we suggest considering pausing treatment with erenumab, fremanezumab, and galcanezumab after 6-12 months of treatment in order to evaluate the migraine status; if migraines recur to the pretreatment status or to an undesirable level for the patient, treatment re-administration is suggested.

- In patients with chronic migraine and medication overuse, we suggest using erenumab, fremanezumab, and galcanezumab before or after withdrawal of acute medications;

- In patients with migraine, we suggest avoiding anti-CGRP monoclonal antibodies in pregnant or nursing women, in individuals with alcohol or drug abuse, cardio and cerebrovascular diseases, and with severe mental disorders;

- In patients with migraine on treatment with anti-CGRP monoclonal antibodies, there is no need for testing for binding and/or neutralizing antibodies.

Adapted from Sacco et al., 2019 [6] with changes 
prevention of EM, with emphasis to topiramate, valproate, flunarizine and venlafaxine. Topiramate $100 \mathrm{mg} / \mathrm{d}$ has little documentation for CM [31, 32]. In addition it can be used as a stabiliser for mood disorders [33] like valproate [34], but the latter has, or should have, very limited use in young females [35]. Venlafaxine is a typical antidepressant and affective disorders are very often comorbid with chronic migraine and/or $\mathrm{MOH}$ [12], thus its use could be helpful when anxiety and/or depressive symptoms are present in patients with CM. Flunarizine showed efficacy in CM in one small size, open label, trial [36]. No combination of topiramate and propranolol is recommended for the prophylactic treatment of $\mathrm{CM}$ [37]. However, botulinum toxin type A (BTXA) and the anti-CGRP mAbs share the best documentation for the prevention of CM (level A). Following the EHF recommendations $[6,7]$, the HHS suggests the use either of BTXA, or anti-CGRP mAbs (erenumab, fremanezumab, or galcanezumab), for those patients who have tried two previous prophylactic treatments for CM (topiramate and flunarizine and/or venlafaxine when depression like symptoms are detected), but they did not tolerate them, or their migraines did not improve. For the use of antiCGRP mAbs and BTXA in CM see Tables 4 and 5, respectively. Both drug classes are safe and well tolerated [38-40]. Notably, the documentation of the first line suggested prophylactic treatments for $\mathrm{CM}$ is very poor or even empirical. However, the Greek Authorities demand failure to first line prophylactic treatments to reimburse BTXA. In order for the Greek doctors to comply with this requirement, they may use topiramate $(100-200 \mathrm{mg} / \mathrm{d})$, flunarizine $(10 \mathrm{mg} / \mathrm{d})$ or venlafaxine $(150 \mathrm{mg} / \mathrm{d})$ as first line treatments.

Table 5 Use of botulinum toxin A (BTXA) in chronic migraine

- BTXA should be administered according to the PREEMPT injection protocol, i.e. injecting $155 \mathrm{U}-195 \mathrm{U}$ to $31-39$ sites every 12-weeks

- Treatment with BTXA should be stopped, if the patient does not respond to the first three treatment cycles.

- Patients treated with BTXA are defined as non-responders, if they have less than $30 \%$ reduction in headache days per month during treatment.

- Evaluate the response to continued treatment with BTXA on the basis of headache calendars by comparing the 4 weeks before and 4 weeks after each treatment cycle.

- Stop treatment in patients with a reduction to less than 10 headache days per month for 3 months (other factors such as headache intensity, disability and patient preferences should also be considered).

- Re-evaluate 4-5 months after stopping BTXA to secure that the patient has not returned to $\mathrm{CM}$.

- In patients with chronic migraine and medication overuse, we suggest using BTXA, before or after withdrawal of acute medications, as second-line prophylactic treatment

Adapted from Bendtsen et al., 2018 [7] with changes
Table 6 Management of Medication Overuse Headache (MOH)

- For decision-making physicians should take into account the patients' preferences, life-style particularities and personal expectancies.

- Physicians should educate patients for the mechanisms underlying the pathophysiology of $\mathrm{MOH}$ and the ways the patients should avoid overusing medications for treating their migraines.

- Depending on the patients' personality, individual preferences and comorbidity, acute withdrawal or tapering down of the drug overuse is recommended.

- Immediate prophylactic treatment together with the drug overuse withdrawal or tapering down, is recommended as well, including topiramate 100-200 mg/day and prednisone or prednisolone $60 \mathrm{mg} / \mathrm{d}$. The use of botulinumtoxinA and/or anti-CGRP monoclonal antibodies (erenumab, fremanezumab, and galcanezumab) is recommended, as second-line treatment.

- Not only migraine, but all potential comorbidities should also be treated.

- To treat anxiety and/or depression like symptoms that are highly likely to co-occur with $\mathrm{MOH}$, venlafaxine $150 \mathrm{mg} / \mathrm{df}$ is recommended.

- Inpatient withdrawal therapy may be needed, in particular for patients overusing opioids, benzodiazepines, or barbiturates.

- Because the prognosis of $\mathrm{MOH}$ is poor and the relapse rate high, close follow-up of patients is recommended.

\section{Medication overuse headache}

Medication overuse headache ( $\mathrm{MOH})$ occurs by definition in chronic migraine [11], although drug overuse may occur in patients suffering from high frequency episodic migraine as well. Prevention of migraine chronification stands as the most important issue in migraine management, as stated previously. As soon as $\mathrm{MOH}$ appears immediate treatment is required, which includes patient education to reduce medication intake, discontinuation of the overused medication and individual prophylactic treatment $[9,41]$. Whether abrupt withdrawal or tapering down of overused medication is better depends on the patient's personality and potential comorbidities he/she may suffer from and it is not related to outcome [9]. Inpatient withdrawal therapy may be needed, in particular for patients overusing opioids, benzodiazepines, or barbiturates [9] (Table 6).

There is moderate evidence for the prophylactic treatment in patients with $\mathrm{CM}$ and medication overuse with topiramate up to $200 \mathrm{mg}$ [9]. Corticosteroids (at least 60 $\mathrm{mg}$ prednisone or prednisolone) and amitriptyline (up to $50 \mathrm{mg}$ ) are possibly effective in the treatment of withdrawal symptoms [9]. However, prophylactic treatment with BTXA and anti-CGRP mAbs also improve $\mathrm{MOH}$ and should be suggested [6, 7] (Tables 4 and 5) in patients who do not need hospitalization. Not only migraine, but all potential comorbidities should be evaluated and treated accordingly to prevent from $\mathrm{MOH}$ recurrence. Because anxiety and depression like symptoms are very common in patients overusing drugs for symptomatic treatment of migraine [12], treatment with venlafaxine $150 \mathrm{mg} / \mathrm{d}$ that also improves both TTH $[42,43]$ and migraine may be 
helpful. Non-pharmaceutical interventions, including cognitive behavioral treatment are also recommended. A large proportion of patients suffering from $\mathrm{MOH}$ often relapse [41] thus close follow-up is recommended.

\section{Neurostimulation for migraine treatment}

Neurostimulation is a novel promising therapeutic approach that helps patients with migraine. Patients in Greece prefer this approach in particular [10] and treating physicians should be aware of these techniques that are divided into invasive and non-invasive approaches. Here we discuss the application only of the non-invasive devices, since the invasive ones require management in a tertiary headache center exclusively [44], such as the vagus nerve stimulation (VNS), the external trigeminal nerve stimulation (eTNS), and the transcranial magnetic stimulation (TMS). In Europe several devices carry the CE mark, among them only four devices have low to moderate evidence for treatment of migraines [45]. The device of the VNS for the symptomatic treatment of migraine [46], the single transcranial magnetic stimulation (sTMS) for the symptomatic treatment of migraine with aura, the repetitive transcranial magnetic stimualtion (rTMS) for the prophylactic treatment of episodic migraine [47] and the eTNS for both the symptomatic and prophylactic treatment of episodic migraine [48, 49]. Thus, migraineurs may use these devices although they are not reimbursed in Greece currently.

\section{Dietary supplements for migraine treatment}

Several randomized clinical trials (RCTs) suggest dietary and vitamin supplementation and different herbs effectiveness in the treatment of migraine, although several methodological shortcomings, especially lack of comparison with standard treatment and placebo, have been noticed [50]. Coenzyme Q10, magnesium citrate, petasites and riboflavin share low to moderate documentation and could be used for migraine prevention when patient preferences exclude pharmacotherapy. There is limited evidence that prophylaxis with combination of simvastatin $(40 \mathrm{mg} / \mathrm{d})$ with vitamin D (1000 Units/d) may help patients suffering from episodic migraine [51]. Other combinations may be useful as well, but the evidence for efficacy is limited. When a patient refuses pharmacotherapy, in particular those patients with negative previous experiences or expectations, e.g. patients with nocebo behaviours [52, 53], HHS suggests the use of dietary supplements to improve migraines. Cognitive behavioural treatment is also effective, not only in these patients, but in those co-suffering from any affective disorder as well [54].

\section{Synopsis}

Migraine is a disabling disease requiring effective treatment. Diagnosis of migraine remains clinical and only in a few cases testing is needed to exclude secondary headaches. HHS suggests taking the interview carefully, based in a semi-structured questionnaire, to cover all aspects of headache features and to follow-up patients with headache diaries. Specific scales to assess headache burden and comorbidities are also suggested, along with a specifically designed database for headache follow-up. For therapeutic decision making physicians should take into account the individual migraine impact and/or disability, potential comorbidities and patient's personal preferences. HHS expresses two major suggestions: adding a rescue medication in the symptomatic migraine treatment and introducing early prophylactic treatment to prevent migraine chronification and medication overuse. For mild migraines, symptomatic treatment with high dose simple analgesics is suggested, while for mild to severe migraines triptans or NSAIDs, or both, should be administered following an individual tailored therapeutic strategy. Rescue medications should be administered parentally (e.g. sumatriptan subcutaneously). For the prevention of episodic migraine, metoprolol $(50-200 \mathrm{mg} /$ d), propranolol $(40-240 \mathrm{mg} / \mathrm{d})$, flunarizine $(5-10 \mathrm{mg} / \mathrm{d})$, valproate $(500-1800 \mathrm{mg} / \mathrm{d})$, topiramate $(25-100 \mathrm{mg} / \mathrm{d})$ and candesartan $(16-32 \mathrm{mg} / \mathrm{d})$ are the drugs of first choice. For chronic migraine prevention topiramate (100$200 \mathrm{mg} / \mathrm{d})$, valproate $(500-1800 \mathrm{mg} / \mathrm{d})$, flunarizine $(5-10$ $\mathrm{mg} / \mathrm{d})$ and venlafaxine $(150 \mathrm{mg} / \mathrm{d})$ may be used, although the evidence is very limited. Botulinum toxin type A and anti-CGRP $m A$ Abs are recommended for patients suffering from chronic migraine (with or without medication overuse) who failed or did not tolerate two previous first choice treatments. Anti-CGRP mAbs are also suggested for patients suffering from high frequency episodic migraine who failed or did not tolerate two previous first choice treatments. Because many migraine sufferers prefer treatment with neurostimulation in Greece, treating physicians should be familiar with these techniques. There is limited but promising evidence for the use of VNS for the symptomatic treatment of migraine, sTMS for the symptomatic treatment of migraine with aura, rTMS for the prophylactic treatment of episodic migraine and eTNS for both the symptomatic and prophylactic treatment of episodic migraine. Dietary supplements may be useful for those refusing pharmacotherapy. Almost all patients would benefit from cognitive behavioural treatment, but only those who prefer and withstand it are good candidates. Safety issues and patients' adherence is of great importance in treatment monitoring. Overall, migraine is a treatable disease that needs particular care. Patients may benefit only if treated appropriately after medical consultation. For refractory cases physicians may refer their patients to the Headache Centres established by the HHS.

\section{Abbreviations}

Anti-CGRP mAbs: Monoclonal antibodies targeting the CGRP pathway; BTXA: Botulinum toxin type A; CGRP: Calcitonin gene-related peptide; 
CM: Chronic Migraine; EFNS: European Federation of Neurological Societies; EHF: European Headache Federation; EP: Episodic Migraine; eTNS: External trigeminal nerve stimulation; HHS: Hellenic Headache Society; HIT6: Headache Impact Test; ICHD-3: International Classification of Headache Disorders-3; mAbs: Monoclonal antibodies; MIDAS: Migraine Disability Assessment Test; MOH: Medication overuse headache; NSAIDs: Non-steroidal anti-inflammatory drugs; RCTs: Randomized clinical trials; TMS: Transcranial magnetic stimulation; TTH: Tension-type headache; VAS: Visual analogue scale fro pain; VNS: Vagus nerve stimulation

\section{Acknowledgements}

There are no acknowledgments.

\section{Declarations}

This article is a Consensus Article from experts in the topic appointed by the Hellenic Headache Society to support Greek physicians who treat headaches.

\section{Authors' contributions}

EK participated in the conception, the design of the work, the acquisition, analysis, interpretation of data and has drafted the manuscript. CA participated in the conception, the design of the work, the acquisition, analysis, interpretation of data and reviewed the manuscript. TSC participated in the conception, the design of the work, the acquisition, analysis, interpretation of data and reviewed the manuscript. EG participated in the conception, the design of the work, the acquisition, analysis, interpretation of data and reviewed the manuscript; NF participated in the conception, the design of the work, the acquisition, analysis, interpretation of data and reviewed the manuscript; TK participated in the conception, the design of the work, the acquisition, analysis, interpretation of data and reviewed the manuscript; EK participated in the conception and reviewed the manuscript; ND participated in the acquisition, analysis, interpretation of data and has drafted the manuscript; DDM participated in the conception, the design of the work, the acquisition, analysis, interpretation of data, has drafted and reviewed the manuscript; All authors have approved the submitted version (and any substantially modified version that involves the author's contribution to the study); and have agreed both to be personally accountable for the author's own contributions and to ensure that questions related to the accuracy or integrity of any part of the work, even ones in which the author was not personally involved, are appropriately investigated, resolved, and the resolution documented in the literature.

\section{Funding}

This work was not founded. The Hellenic Headache Society covered the publication fees.

\section{Availability of data and materials}

There are no original data.

\section{Ethics approval and consent to participate}

Ethics approval and consent to participate was not needed for this consensus.

\section{Consent for publication}

All authors have reviewed the final version and gave their approval for publication.

\section{Competing interests}

- Dr. Evangelos Kouremenos has received advisory fees and travel grants from Novartis, Merck, Sanofi, Genesis, TEVA, Specifar, Pharmathen and Innovis.

- Dr. Chrysa Arvaniti has received honoraria and travel grants from Novartis, GlaxoSmithKlin and Teva pharmaceuticals.

- Dr. Theodoros S Constantinidis has received advisory fees, honoraria, research grants, or travel grants from Allergan; Bayer; Braintherapeutics; C.A. Papaellinas, Elpen, Genesis Pharma; Merck, Novartis; Pharmathen; Roche; Sanofi-Genzyme; Teva Pharmaceuticals; and UCB. He is the current vice-president of the Hellenic Headache Society and has no ownership interests and does not own any pharmaceutical company stocks.

- Dr. Ermioni Giannouli has no disclosures.
- Dr. Nikolaos Fakas has received consultation, speech honoraria, trave grants, clinical research support from Actelion; AB Science; Allergan; Amgen; Bayer; Biogen; Celgene; Genesis Pharma; Roche; Eli Lilly; Merck-Serono; Mylan; Novartis; Receptos; Sanofi-Genzyme; and Teva;

- Dr. Themistoklis Kalamatas has no disclosures.

- Prof. Evangelia Kararizou has received advisory fees, or travel grants from Genesis Pharm; Novartis; Sanofi-Genzyme; and Teva Pharmaceuticals.

- Dr. Dimitrios Naoumis has received advisory fees and travel grants from Novartis, Merck, Sanofi, Genesis, TEVA, Specifar, Pharmathen and Innovis.

- Prof. Dimos D. Mitsikostas has received advisory fees, honoraria, research grants, and/or travel grants from Allergan; Amgen; Biogen; Cefaly; electroCore, Inc.; Genesis Pharma; Eli Lilly and Company; Merz Pharma; Mylan; Novartis; Roche; Sanofi Genzyme; and Teva Pharmaceuticals. He has been the primary investigator for Amgen, electroCore, Eli Lilly and Company, Novartis, and Teva trials. DDM is the current president of the Hellenic Headache Society and has no ownership interests and does not own any pharmaceutical company stocks.

\section{Author details}

${ }^{1}$ Neurology Department, 251 Air Force General Hospital, Athens, Greece. ${ }^{2}$ Second Neurology Department, School of Medicine, National \& Kapodistrian University of Athens, Attikon Hospital, Athens, Greece. ${ }^{3}$ Private Headache Office, Korinthos, Greece. ${ }^{4}$ Department of Neurology, Athens Medical Center, Athens, Greece. ${ }^{5}$ Neurology Department, 401 Military General Hospital, Athens, Greece. ${ }^{6}$ First Neurology Department, School of Medicine, National \& Kapodistrian University of Athens, Aeginition Hospital, 72-74 VI Sofia's Avenue, 11528 Athens, Greece.

\section{Received: 20 September 2019 Accepted: 18 November 2019}

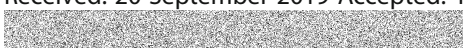

\section{References}

1. GBD 2016 Headache Collaborators (2018) Global, regional, and national burden of migraine and tension-type headache, 1990-2016: a systematic analysis for the Global Burden of Disease Study 2016. Lancet Neurol 17:954-976

2. Steiner T, Lars J, Stovner LJ, Theo Vos T, Jensen R, Katsarava Z (2018) Migraine is first cause of disability in under 50s: will health politicians now take notice? J Headache Pain 19:17-20

3. Linde M, Gustavsson A, Stovner LJ, Steiner TJ, Barré J, Katsarava Z, Lainez JM, Lampl C, Lantéri-Minet M, Rastenyte D, Ruiz de la Torre E, Tassorelli C, Andrée C (2012) The cost of headache disorders in Europe: the Eurolight project. Eur J Neurol 19:703-711

4. Mitsikostas DD, Arvaniti C, Constantinidis T, Fakas N, Dermitzakis E, Rudolf J, Vikelis M (2018) A population based survey for headaches in Greece to estimate prevalence and treatment preferences. Neurology 90(Suppl 15):P3.143

5. Mitsikostas DD, Ashina M, Craven A, Diener HC, Goadsby PJ, Ferrari MD, Lampl C, Paemeleire K, Pascual J, Siva A, Olesen J, Osipova V, Martelletti P, EHF committee (2015) European Headache Federation consensus on technical investigation for primary headache disorders. J Headache Pain 17:5

6. Sacco S, Bendtsen L, Ashina M, Reuter U, Terwindt G, Mitsikostas DD, Martelletti P (2019) European headache federation guideline on the use of monoclonal antibodies acting on the calcitonin gene related peptide or its receptor for migraine prevention. J Headache Pain. 20:6

7. Bendtsen L, Sacco S, Ashina M, Mitsikostas D, Ahmed F, Pozo-Rosich P, Martelletti P (2018) Guideline on the use of onabotulinumtoxinA in chronic migraine: a consensus statement from the European Headache Federation. $\checkmark$ Headache Pain 19:91

8. Evers S, Afra J, Frese A, Goadsby PJ, Linde M, May A, Sándor PS, European Federation of Neurological Societies (2009) EFNS guideline on the drug treatment of migraine-revised report of an EFNS task force. Eur J Neurol 16:968-981

9. Evers S, Jensen R, European Federation of Neurological Societies (2011) Treatment of medication overuse headache--guideline of the EFNS headache panel. Eur J Neurol 18:1115-1121 
10. Mitsikostas DD, Belesioti I, Arvaniti C, Mitropoulou E, Deligianni C, Kasioti E, Constantinidis T, Dermitzakis M, Vikelis M, Hellenic Headache Society (2017) Patients' preferences for headache acute and preventive treatment. J Headache Pain 18:102

11. Headache Classification Committee of the International Headache Society (IHS) (2013) The international classification of headache disorders, 3rd edition (beta version). Cephalalgia 33:629-808

12. Mitsikostas DD, Thomas A (1999) Comorbidity of headache and depressive disorders. Cephalalgia 19:211-217

13. Oikonomidi T, Vikelis M, Artemiadis A, Chrousos GP, Darviri C (2018) Reliability and validity of the Greek migraine disability assessment (MIDAS) questionnaire. Pharmacoecon Open 2:77-85

14. Kavasis I, Samakouri M, Mallis D, Terzoudi A, Papanastassiou E, Tzavaras N, Livaditis M (2005) Translation and validation of the depression outcomes module (DOM) in Greece. Int J Methods Psychiatr Res 14:221-229

15. Bjorner JB, Kosinski M, Ware JE Jr (2003) Calibration of an item pool for assessing the burden of headaches: an application of item response theory to the headache impact test (HIT). Qual Life Res 12:913-933

16. Mitsikostas DD, Vikelis M, Kodounis A, Zaglis D, Xifaras M, Doitsini S, Georgiadis G, Thomas A, Charmoussi S (2010) Migraine recurrence is not associated with depressive or anxiety symptoms. Results of a randomized controlled trial. Cephalalgia. 30:690-695

17. Ferrari MD, James MH, Bates D et al (1994) Oral sumatriptan: effect of a second dose, and incidence and treatment of headache recurrences. Cephalalgia 14:330-338

18. Brandes $J$, Kudrow D, Stark SR, O'Carroll CP, Adelman JU, O'Donnell FJ, Alexander WJ, Spruill SE, Barrett PS, Lener SE (2007) Sumatriptan-naproxen for acute treatment of migraine: a randomized trial. JAMA. 297:1443-1454

19. Silberstein SD, Mannix LK, Goldstein J, Couch JR, Byrd SC, Ames MH, McDonald SA, Lener SE, Toso C (2008) Multimechanistic (sumatriptannaproxen) early intervention for the acute treatment of migraine. Neurology. 71:114-121

20. Calhoun AH, Ford S (2014) Double-blind, placebo-controlled, crossove study of early-intervention with sumatriptan 85/naproxen sodium 500 in (truly) episodic migraine: what's neck pain got to do with it? Postgrad Med 126:86-90

21. Thorlund K, Toor K, Wu P, Chan K, Druyts E, Ramos E, Bhambri R, Donnet A, Stark R, Goadsby PJ (2017) Comparative tolerability of treatments for acute migraine: A network meta-analysis. Cephalalgia. 37:965-978

22. Engindeniz Z, Demircan C, Karli N, Armagan E, Bulut M, Aydin T, Zarifoglu M (2005) Intramuscular tramadol vs. diclofenac sodium for the treatment of acute migraine attacks in emergency department: a prospective, randomised, double-blind study. J Headache Pain. 6:143-148

23. Mazaheri S, Poorolajal J, Hosseinzadeh A, Fazlian MM (2015) Effect of intravenous sodium valproate vs dexamethasone on acute migraine headache: a double blind randomized clinical trial. PLoS One 10:e0120229

24. Silberstein SD, Freitag FG, Rozen TD, Hewitt DJ, Jordan DM, Fisher AC, Rosenthal NR (2005) Tramadol/acetaminophen for the treatment of acute migraine pain: findings of a randomized, placebo-controlled trial. Headache 45:1317-1327

25. Doğan NÖ, Pekdemir M, Yılmaz S, Yaka E, Karadaş A, Durmuş U, Avcu N, Koçkan E (2019) Intravenous metoclopramide in the treatment of acute migraines: A randomized, placebo-controlled trial. Acta Neurol Scand 139:334-339

26. Kirthi V, Derry S, Moore RA (2013) Aspirin with or without an antiemetic for acute migraine headaches in adults. Cochrane Database Syst Rev (4): CD008041. https://doi.org/10.1002/14651858.CD008041.pub3

27. Orr SL, Friedman BW, Christie S, Minen MT, Bamford C, Kelley NE, Tepper D (2016) Management of Adults with Acute Migraine in the emergency department: the American headache society evidence assessment of parenteral pharmacotherapies. Headache. 56:911-940

28. Deligianni Cl, Vikelis M, Mitsikostas DD (2012) Depression in headaches: chronification. Curr Opin Neurol 25:277-283

29. Tronvik E, Stovner L, Helde G, Sand T, Bovim G (2003) Prophylactic treatment of migraine with an angiotensin II receptor blocker: a randomized controlled trial. JAMA. 289:65-69

30. Stovner LJ, Linde M, Gravdahl GB, Tronvik E, Aamodt AH, Sand T, Hagen K (2014) A comparative study of candesartan versus propranolol for migraineprophylaxis: A randomised, triple-blind, placebo-controlled, double cross-over study. Cephalalgia. 34:523-532

31. Silberstein S, Lipton R, Dodick D, Freitag F, Mathew N, Brandes J, Bigal M, Ascher S, Morein J, Wright P, Greenberg S, Hulihan J (2009) Topiramate treatment of chronic migraine: a randomized, placebo-controlled trial of quality of life and other efficacy measures. Headache. 49:1153-1162

32. Lipton RB, Silberstein S, Dodick D, Cady R, Freitag F, Mathew N, Biondi DM, Ascher S, Olson WH, Hulihan J (2011) Topiramate intervention to prevent transformation of episodic migraine: the topiramate INTREPID study. Cephalalgia. 31:18-30

33. Mowla A, Kardeh E (2011) Topiramate augmentation in patients with resistant major depressive disorder: a double-blind placebo-controlled clinical trial. Prog Neuro-Psychopharmacol Biol Psychiatry 35:970-973

34. Peselow ED, Clevenger S, IsHak WW (2016) Prophylactic efficacy of lithium, valproic acid, and carbamazepine in the maintenance phase of bipolar disorder: a naturalistic study. Int Clin Psychopharmacol 31:218-223

35. Bosak M, Słowik A, Turaj W (2019) Why do some women with epilepsy use valproic acid despite current guidelines? A single-center cohort study. Epilepsy Behav 98:1-5

36. Lai KL, Niddam DM, Fuh JL, Chen SP, Wang YF, Chen WT, Wu JC, Wang SJ (2017) Flunarizine versus topiramate for chronic migraine prophylaxis: a randomized trial. Acta Neurol Scand 135:476-483

37. Silberstein SD, Dodick DW, Lindblad AS, Holroyd K, Harrington M, Mathew NT, Hirtz D, Chronic Migraine Treatment Trial Research Group (2012) Randomized, placebo-controlled trial of propranolol added to topiramate in chronic migraine. Neurology 78:976-984

38. Mitsikostas DD, Reuter U (2017) Calcitonin gene-related peptide monoclonal antibodies for migraine prevention: comparisons across randomized controlled studies. Curr Opin Neurol 30:272-280

39. Mitsikostas DD, Rapoport AM (2015) New players in the preventive treatment of migraine. BMC Med 13:279

40. Diener HC, Dodick DW, Turkel CC, Demos G, Degryse RE, Earl NL, Brin MF (2014) Pooled analysis of the safety and tolerability of onabotulinumtoxinA in the treatment of chronic migraine. Eur J Neurol 21:851-859

41. Diener HC, Dodick D, Evers S, Holle D, Jensen RH, Lipton RB, Porreca F, Silberstein S, Schwedt T (2019) Pathophysiology, prevention, and treatment of medication overuse headache. Lancet Neurol 18:891-902

42. Zissis NP, Harmoussi S, Vlaikidis N, Mitsikostas D, Thomaidis T, Georgiadis G, Karageorgiou K (2007) A randomized, double-blind, placebo-controlled study of venlafaxine XR in out-patients with tension-type headache. Cephalalgia. 27:315-324

43. Bendtsen L, Evers S, Linde M, Mitsikostas DD, Sandrini G, Schoenen J, EFNS (2010) EFNS guideline on the treatment of tension-type headache - report of an EFNS task force. Eur J Neurol 17:1318-1325

44. Martelletti $P$, Jensen $\mathrm{RH}$, Antal A, Arcioni R, Brighina F, de Tommaso M, Franzini $A$, Fontaine $D$, Heiland $M$, Jürgens TP, Leone $M$, Magis $D$, Paemeleire K, Palmisani S, Paulus W, May A, European Headache Federation (2013) Neuromodulation of chronic headaches: position statement from the European Headache Federation. J Headache Pain 14:86

45. Reuter U, McClure C, Liebler E, Pozo-Rosich P (2019) Non-invasive neuromodulation for migraine and cluster headache: a systematic review of clinical trials. J Neurol Neurosurg Psychiatry 90:796-804

46. Tassorelli C, Grazzi L, de Tommaso M, Pierangeli G, Martelletti P, Rainero I, Dorlas S, Geppetti P, Ambrosini A, Sarchielli P, Liebler E, Barbanti P, PRESTO Study Group (2018) Non-invasive vagus nerve stimulation as acute therapy for migraine: The randomized PRESTO study. Neurology. 91:e364-e373

47. Stilling JM, Monchi O, Amoozegar F, Debert CT (2019) Transcranial Magnetic and Direct Current Stimulation (TMS/tDCS) for the Treatment of Headache: A Systematic Review. Headache. 59:339-357

48. Chou DE, Shnayderman Yugrakh M, Winegarner D, Rowe V, Kuruvilla D, Schoenen J (2019) Acute migraine therapy with external trigeminal neurostimulation (ACME): a randomized controlled trial. Cephalalgia. 39:3-14

49. Schoenen J, Vandersmissen B, Jeangette $S$, Herroelen L, Vandenheede M, Gérard P, Magis D (2013) Migraine prevention with a supraorbital transcutaneous stimulator: a randomized controlled trial. Neurology. 80 : 697-704

50. Rehman T, Ahmad S, Fatima Q (2019) Effects of dietary supplementations and herbs on migraine - a systematic review. J Complement Integr Med. 16(3). https://doi.org/10.1515/jcim-2018-0143

51. Buettner C, Nir RR, Bertisch SM, Bernstein C, Schain A, Mittleman MA, Burstein R (2015) Simvastatin and vitamin D for migraine prevention: A randomized, controlled trial. Ann Neurol 78:970-981

52. Mitsikostas DD, Mantonakis LI, Chalarakis NG (2011) Nocebo is the enemy, not placebo. A meta-analysis of reported side effects after placebo treatment in headaches. Cephalalgia. 31:550-561 
53. Mitsikostas DD (2016) Nocebo in headache. Curr Opin Neurol 29:331-336

54. Lee HJ, Lee JH, Cho EY, Kim SM, Yoon S (2019) Efficacy of psychological treatment for headache disorder: a systematic review and meta-analysis. J Headache Pain. 20:17

\section{Publisher's Note}

Springer Nature remains neutral with regard to jurisdictional claims in published maps and institutional affiliations.

Ready to submit your research? Choose BMC and benefit from:

- fast, convenient online submission

- thorough peer review by experienced researchers in your field

- rapid publication on acceptance

- support for research data, including large and complex data types

- gold Open Access which fosters wider collaboration and increased citations

- maximum visibility for your research: over $100 \mathrm{M}$ website views per year

At $B M C$, research is always in progress.

Learn more biomedcentral.com/submissions 